\title{
Protective Effect of Ginsenoside against Acute Renal Failure and Expression of Tyrosine Hydroxylase in the Locus Coeruleus
}

\author{
H. A. ZHANG ${ }^{1}$, M. WANG ${ }^{1}$, J. ZHOU ${ }^{1}$, Q. Y. YAO ${ }^{1}$, J. M. MA ${ }^{2}$, C. L. JIANG ${ }^{1}$ \\ ${ }^{1}$ Department of Physiology, Dalian Medical University, Dalian, China, ${ }^{2}$ Department of Anatomy, \\ Dalian Medical University, Dalian, China
}

Received July 18, 2008

Accepted January 19, 2009

On-line February 27, 2009

\begin{abstract}
Summary
Acute renal failure (ARF) is mainly characterized by acute tubular necrosis. No significant change was found for mortality rates over the past few decades despite significant advances in supportive care. In recent years, great effort has been focused on traditional and herbal medicine, which is much less toxic than those agents conventionally used and which is nowadays considered as a novel therapeutic agent for ARF. However, the effect of ginsenosides (GS) administered orally on ARF has not been reported yet and little is known about its cellular and molecular mechanism. The purpose of the study is to investigate the protective effect of ginsenoside in rats with ARF on the changes of tyrosine hydroxylase immunoreactivity (TH-IR) as well as on the involvement of mitogen-activated protein kinases (MAPK) in the locus coeruleus. In our assay, glycerol-induced acute renal failure in rats was employed to study the protective effects of ginsenoside. Our results indicated that the treatment of ARF rats with ginsenosides for $48 \mathrm{~h}$ significantly reduced the serum blood urea nitrogen, creatinine level, and lipid peroxidation, restored the GSH level and the normal renal morphology. Immunohistochemistry showed that an obvious increase of TH-IR was further enhanced in ARF+GS group. The same effect was also observed in the changes of p-ERK1/2-IR in the locus coeruleus. Our results suggest that ginsenoside administered orally may have a strong renal protective effect against glycerol-induced ARF, and ginsenoside can also activate the brain catecholaminergic neurons in the locus coeruleus. Our future attention will be focused to the question whether there is a correlation between the renal protective effect of ginsenosides against acute renal failure and the activation of tyrosine hydroxylase in the locus coeruleus.
\end{abstract}

\section{Key words}

Ginsenoside • Acute renal failure $\bullet$ Locus coeruleus $\bullet \cdot$ Tyrosine hydroxylase $\bullet$ Protective effect

\section{Corresponding author}

C. L. Jiang, Department of Physiology, Dalian Medical University, Dalian, Liaoning, China. E-mail: chunling_jiang2006@ yahoo.com.cn

\section{Introduction}

Acute renal failure (ARF) is frequent in hospitalized critically ill patients and mortality associated with ARF is largely unchanged over many decades. ARF is mainly characterized by acute tubular necrosis. Progress in elucidation of ARF pathophysiology has led to the development and testing of many therapeutic drugs and other interventions in animal and human forms of acute tubular necrosis (Kellum 2004, Lameire et al. 2003). Renal replacement therapy has promising features in treating of ARF, especially before complications. However, it was reported that the incidence of ARF was rising over the past two decades (Hou et al. 1983, Nash et al. 2002) and mortality exceeded $50 \%$ among those who required dialysis support (Metnitz et al. 2002, Mehta et al. 2004). Therefore, mortality rates have changed little over the past few decades despite significant advances in supportive care. Therefore, preventions of the occurrence and progression of ARF has become a very important issue. In recent years, great effort has been focused on traditional and herbal medicine without toxic effects to provide a novel therapeutic agent for ARF. 
Panax ginseng C.A. Meyer is a well-known folk medicine in the Far East countries and it has served as an important component of many Chinese prescriptions since thousands of years (Wen et al. 1996). Ginsenosides (GS), the principal active ingredients of ginseng, have a wide range of pharmacological and physiological actions, such as antiaging, immunoenhancement, antistress and antitumor (Sugaya et al. 1988, Hasegawa et al. 2002, Kaneko et al. 2004). Moreover, it has been reported that sun ginseng (SG, heat-processed Panax ginseng at $120{ }^{\circ} \mathrm{C}$ ) showed strong protective effect against diabetic renal damage (Kang et al. 2006). However, the effect of GS administrated orally on ARF has not been reported yet and little is known about its cellular and molecular mechanism.

Locus coeruleus (LC), an important integrative site in the pons, regulates sympathetic nerve activity, fluid balance and arginine vasopressin (AVP) release. Tyrosine hydroxylase ( $\mathrm{TH})$, the rate-limiting enzyme in catecholamine synthesis, is highly expressed in locus coeruleus in the brain. Exposure to many types of physiological, social or pharmacological stressors, such as cold, restraint, footshock, isolation, forced walking and chronic social stress increases TH mRNA in the LC (Angulo et al. 1991, Watanabe et al.1995, Rusnák et al. 1998). It was reported that the increase in the expression of $\mathrm{TH}$ in the LC coincides with increased transcription (Chang et al. 2000). Similarly, the TH gene is often associated with the induction of AP-1 transcription factor c-Fos (Sun et al. 2003). The mitogen-activated protein kinases (MAPK) are involved in the intracellular signaling pathways, transferring extracellular stimuli into intracellular transcriptional responses. They play an important role in the activation of several transcription factors (Karin et al. 1995). The extracellular signalregulated kinases (ERK) represent one of the four subfamilies of MAP kinases. In particular, ERK stimulate c-fos expression (Whitmarsh et al. 1996), indicating that MAPK may facilitate the formation of c-fos/c-Jun heterodimmers, controlling the regulation of AP-1 activity. We have hypothesized that MAPK might be involved in the differential regulation of $\mathrm{TH}$ induction in the brain catecholaminergic neurons.

A recent in vivo study indicated that the phenotypic differentiation of LC noradrenergic neurons mediated by brain-derived neurotrophic factor (BDNF) was enhanced by corticotrophin-releasing factor (CRF) through the activation of a cAMP-dependent signaling pathway that involved the activation of ERK1/2 (Traver et al.2006). Previous in vitro experiment suggested that ERK signaling pathway in the kidney was strongly related to the renal function and renal cell regeneration after the glycerol injection (Ishizuka et al. 1999). However, the roles of brain catecholaminergic neural pathway and brain MAPK signal pathway, as well as their interaction in glycerol-induced ARF rats remains unclear.

Based on these findings, it could be speculated that ginsenosides might have protective effects and there might be some interactions between catecholaminergic neurons and MAPK signal pathway in the LC of glycerol-induced ARF rats. Therefore, we examined 1) the changes of blood urea nitrogen and serum creatinine in ARF rats treated with ginsenosides for $48 \mathrm{~h}, 2$ ) the changes of malondialdehyde and the reduced glutathione in renal cortex homogenate of ARF rats treated with ginsenoside for $48 \mathrm{~h}, 3$ ) the changes of tyrosine hydroxylase-immunoreactivity in the LC of glycerolinduced ARF rats treated with ginsenoside for $48 \mathrm{~h}$, and 4) the changes of phospho-ERK1/2-immunoreactivity (p-ERK1/2-IR) in the LC of glycerol-induced ARF rats treated with ginsenoside for $48 \mathrm{~h}$.

\section{Methods}

Animals

Healthy male Sprague-Dawley rats (Dalian Medical University Animal Center, China) weighing 180$220 \mathrm{~g}$ were kept on a $12 \mathrm{~h} / 12 \mathrm{~h}$ light/dark schedule with a free access to standard laboratory food and water at room temperature. During this time, rats were handled daily to avoid stress-induced expression on the day of the experiment.

\section{Animal treatment}

Eighty male rats were used in this study. After several days of adaptation, they were deprived of water for $16 \mathrm{~h}$ (from 4 p.m. to 8 a.m.).

Forty rats for the experiment in vivo were divided randomly into four groups ( $\mathrm{n}=10$ per group): acute renal failure (ARF) + physiological saline (NS) group, ARF + ginsenoside (GS) group, NS+NS group and $\mathrm{NS}+\mathrm{GS}$ group. After being deprived of water for $16 \mathrm{~h}$, $\mathrm{ARF}+\mathrm{NS}$ group and ARF+GS group were given intramuscularly $10 \mathrm{ml} / \mathrm{kg}$ b.w. of $50 \%$ (vol/vol) glycerol solution distributed equally in both hind limbs. As soon as the model was established, $\mathrm{ARF}+\mathrm{GS}$ group was given $25 \mathrm{mg}$ GS in $2 \mathrm{ml} \mathrm{NS}$ using a stomach tube, at the same 
time, ARF+NS group was given $2 \mathrm{ml} \mathrm{NS}$. The treatment of the other two groups were analogous. They were treated with GS or NS for two consecutive days (once per $6 \mathrm{~h}$, twice per day).

Another 24 rats for immunohistochemistry were also divided into 4 groups and treated as described in the experiment in vivo.

\section{Experiment in vivo}

After treatment with ginsenoside or physiological saline for $48 \mathrm{~h}$, blood samples for urea nitrogen and creatinine determination were taken from the angulus oculi medialis. Subsequently kidney was removed, then renal cortex was homogenized with icecold physiological saline. Blood urea nitrogen (BUN) was assayed by the Fearon method and serum creatinine (Cre) by the Jaffe method using standard diagnostic kits. The malondialdehyde (MDA) content, a measure of lipid peroxidation, was assayed in the form of thiobarbituric acid reacting substances (TBARS) using commercial reagents (Nanjing Jiancheng Bioengineering Institute, P.R. China). The reduced glutathione (GSH) was measured by the method of Jollow using commercial reagents (Nanjing Jiancheng Bioengineering Institute, P.R. China). It was observed by measuring the absorbance at $412 \mathrm{~nm}$.

\section{Renal histology}

Forty-eight hours after the administration of ginsenoside or physiological saline, 40 rats were deeply anesthetized with $4 \%$ chloral hydrate $(400 \mathrm{mg} / \mathrm{kg}$ b.w., i.p.) and perfused transcardially with $1 \%$ and $4 \%$ paraformaldehyde for the fixation of the brain and kidney tissue. Brain and kidney tissue were removed, post-fixed in $4 \%$ paraformaldehyde and immersed into a phosphate buffer saline (PBS) containing $30 \%$ sucrose for 3 days.

Kidney was washed by pure water for $12 \mathrm{~h}$ and then was embedded in paraffin and used for histological examination. Sections $(4 \mu \mathrm{m}$ thick $)$ were cut, deparaffinized, hydrated and stained with hematoxylin and eosin. The renal sections were examined in blind fashion for hemorrhagic and hyaline casts, tubular necrosis and apical blebbing in all treatments.

\section{Immunohistochemistry analysis}

When the brain tissues were submerged, $50 \mu \mathrm{m}$ thick coronal brain sections were sliced on a vibratome. The identification of locus coeruleus (bregma $-9.68 \mathrm{~mm}$ to $-10.04 \mathrm{~mm}$ ) was based on the atlas by Paxinos and
Watson. The sections above were rinsed three times in PBS for $10 \mathrm{~min}$ and then incubated with $0.2 \%$ Triton for $5 \mathrm{~min}$. Then the sections were rinsed three times in PBS $10 \mathrm{~min}$ and incubated with bovine serum albumin $(2 \%$ BSA) (Sigma Co., USA) for $1 \mathrm{~h}$. Thereafter the sections were incubated with the primary antibody (TH-Ab, 1:1000, Sigma Co., USA; p-ERK1/2-Ab, 1:100, Boster Company, P.R. China) overnight at $4{ }^{\circ} \mathrm{C}$. The sections were then rinsed three times in PBS for $10 \mathrm{~min}$ and incubated with $2 \%$ BSA for $20 \mathrm{~min}$. Subsequently, the sections were rinsed three times in PBS for $10 \mathrm{~min}$, and further incubated with the biotinylated-second antibody (Boster Company, P.R. China) at room temperature for $2 \mathrm{~h}$. Finally, the sections were again rinsed three times in PBS for $10 \mathrm{~min}$ and incubated with the avidin-biotin complex ABC (Boster Company, P.R. China) at room temperature for $2 \mathrm{~h}$. Diaminobenzidine (DAB, Sigma Co., USA) was used for signal detection. The control sections were incubated with PBS instead of primary antibody. The HPIAS (Highresolution pathological image analysis system) series colorful pathology photographic system was used to analyze TH-IR and p-ERK1/2-IR positive neurons. The brain sections were observed in a $20 \times$ magnification. The number and optical density of TH-IR and p-ERK1/2-IR positive neurons were calculated per area and per group.

\section{Statistical analysis}

All data were expressed as mean \pm S.E.M. Statistical evaluation was done using ANOVA with posthoc test of LSD in Equal Variances Assumed. In all comparisons, statistical significance was set at $\mathrm{P}<0.05$.

\section{Results}

\section{Effect of GS on glycerol-induced renal dysfunction}

Glycerol administration resulted in a significant increase in BUN and Cre levels as compared to those in $\mathrm{NS}+\mathrm{NS}$ group. On the contrary, glycerol-induced ARF rats treated with ginsenoside for $48 \mathrm{~h}(\mathrm{ARF}+\mathrm{GS}$ group) showed a significant decrease in BUN and Cre (Fig. 1, $\mathrm{P}<0.05$ ).

\section{Effect of GS on renal MDA and GSH level}

Glycerol-treated rats (ARF+NS group) showed a significant lipid peroxidation as indicated by a marked increase of renal MDA level and decreased GSH level as compared to that in NS+NS group. However, treatment with GS for $48 \mathrm{~h}$ (ARF+GS group) significantly reduced the MDA level and restored the GSH level as compared to $\mathrm{ARF}+\mathrm{NS}$ group (Fig. 2, $\mathrm{P}<0.05$ ). 
A

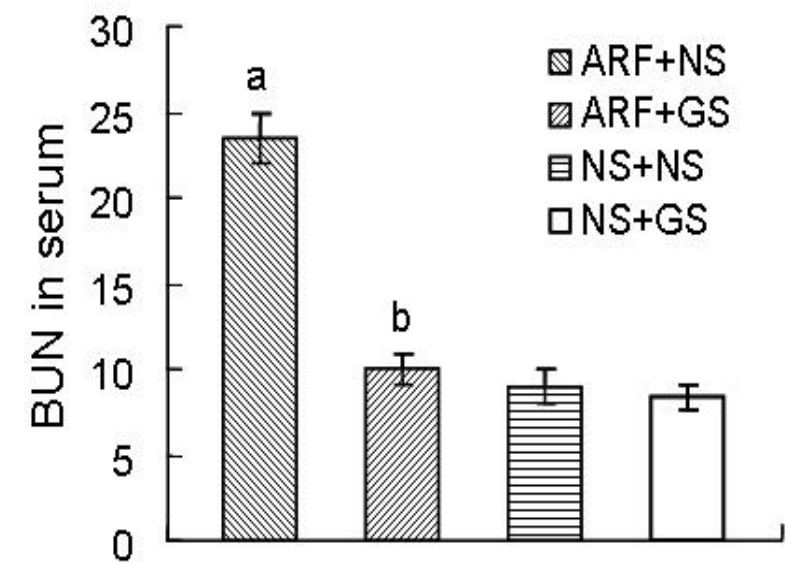

B

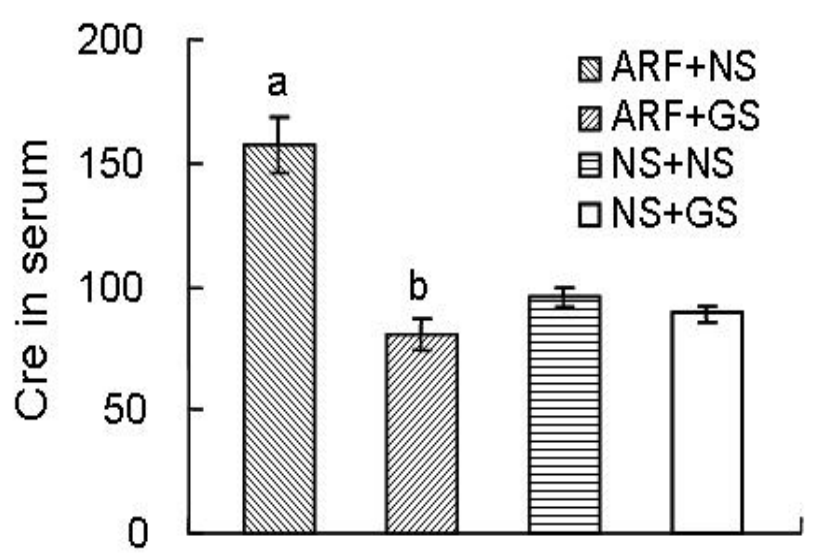

Fig. 1. Changes of BUN and Cre in serum in the ARF rats treated with GS for 48 h. A. BUN in serum B. Cre in serum. Data are mean \pm S.E.M. $n=10$ ( ${ }^{\mathrm{a}} \mathrm{P}<0.05$ ARF+NS group vs. NS+NS group, ${ }^{\mathrm{b}} \mathrm{P}<0.05 \mathrm{ARF}+\mathrm{GS}$ group vs. ARF+NS group).

A

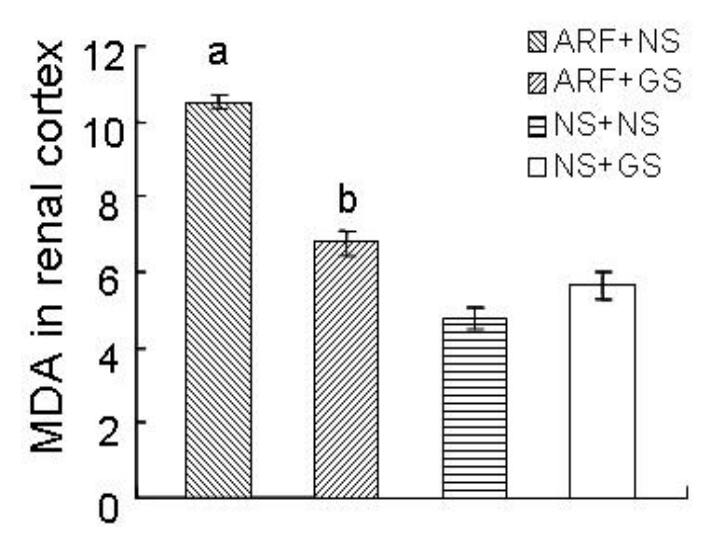

B

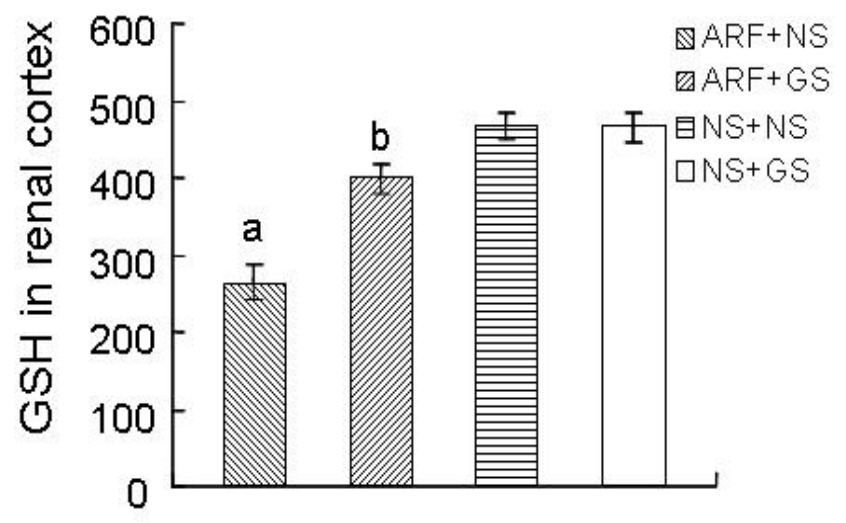

Fig. 2. Changes of MDA and GSH in renal cortex homogenate in the ARF rats treated with GS for $48 \mathrm{~h}$. A. MDA in renal cortex B. GSH in renal cortex. Data are mean \pm S.E.M. $n=10$ ( ${ }^{\mathrm{P}} \mathrm{P}<0.05$ ARF+NS group vs. NS+NS group, ${ }^{\mathrm{b}} \mathrm{P}<0.05$ ARF+GS group vs. ARF+NS group).

Effect of GS on glycerol-induced changes in renal morphology

The histopathological changes were showed in Figure 3. The NS+NS group did not show any morphological changes (Fig. 3C). By contrast, the kidneys of rats treated with glycerol showed marked histological changes in cortex. The renal sections showed severe apical blebbing, hyaline casts and tubular necrosis (Fig. 3A). Kidney sections of GS-treated rats preserved the normal morphology of the kidney (Fig. 3B).

Effect of GS on the changes of TH-IR in the LC of ARF rats induced by glycerol

In the pons of NS+NS group, TH-IR positive neurons were distributed predominantly in the LC (Fig. 4C). The most striking difference in TH-IR positive neurons in ARF+NS group compared with the NS+NS group was a significant increase in optical density and number of TH-IR staining neurons in the LC. This was illustrated by comparing Figure $4 \mathrm{~A}$ (ARF+NS group) with Figure 4C (NS+NS group). There was a further increase of optical density and number of TH-IR positive neurons in the LC of ARF+GS group (Fig. 4B) when compared with ARF+NS group. TH-IR in the LC of NS+GS group was slightly increased, compared with that of NS+NS group (Fig. 5).

Effect of $G S$ on the changes of $p$-ERK1/2-IR in the LC in ARF rats induced by glycerol

Immunohistochemistry also showed an obvious increase of ERK-IR in the LC of ARF+NS group (Fig. 6A, $\mathrm{P}<0.05$ ); but $\mathrm{p}$-ERK-IR $1 / 2$ was further enhanced in $\mathrm{ARF}+\mathrm{GS}$ group compared with $\mathrm{ARF}+\mathrm{NS}$ group (Fig. 6B, $\mathrm{P}<0.05$ (Fig. 7). 

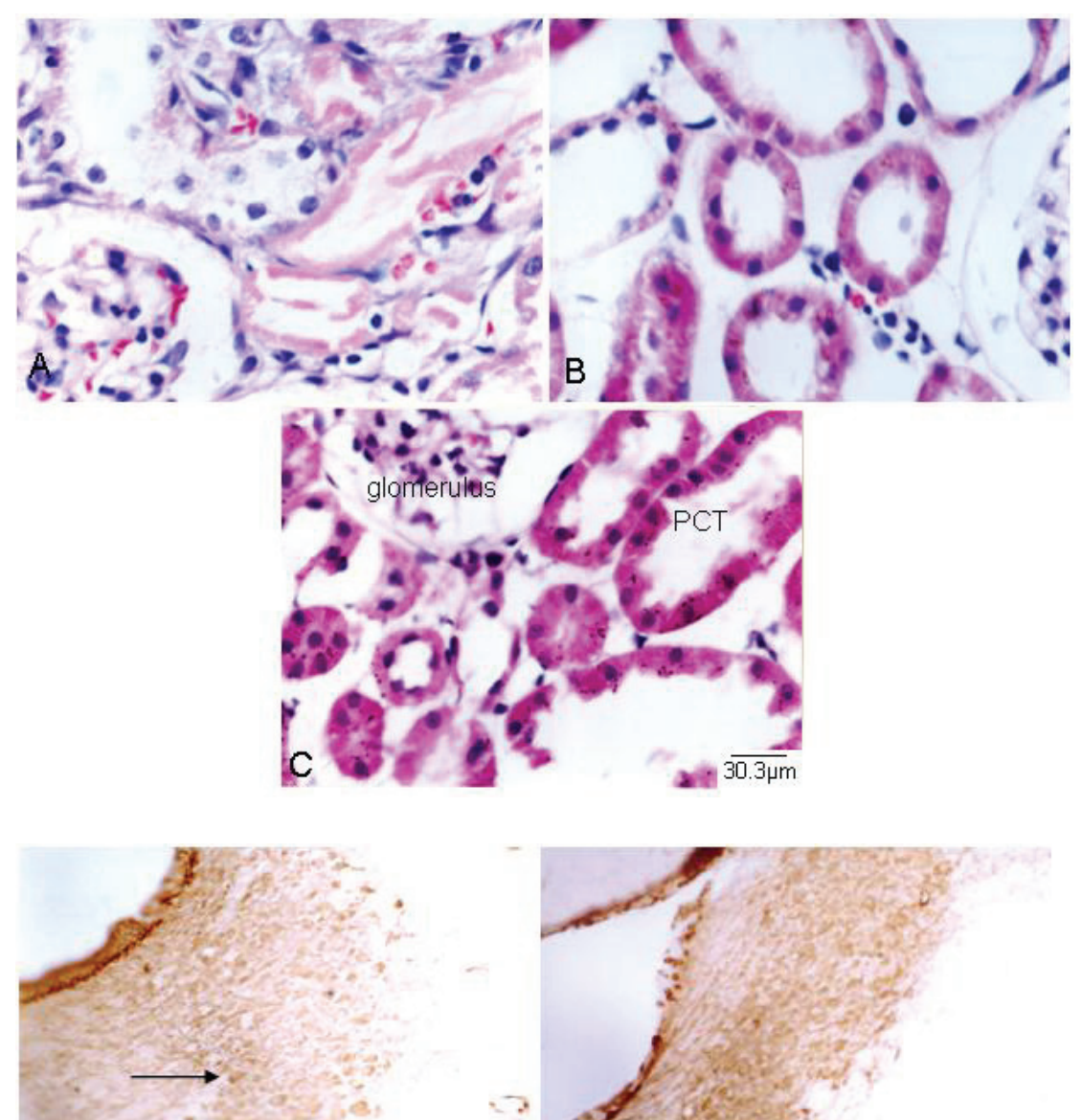

A
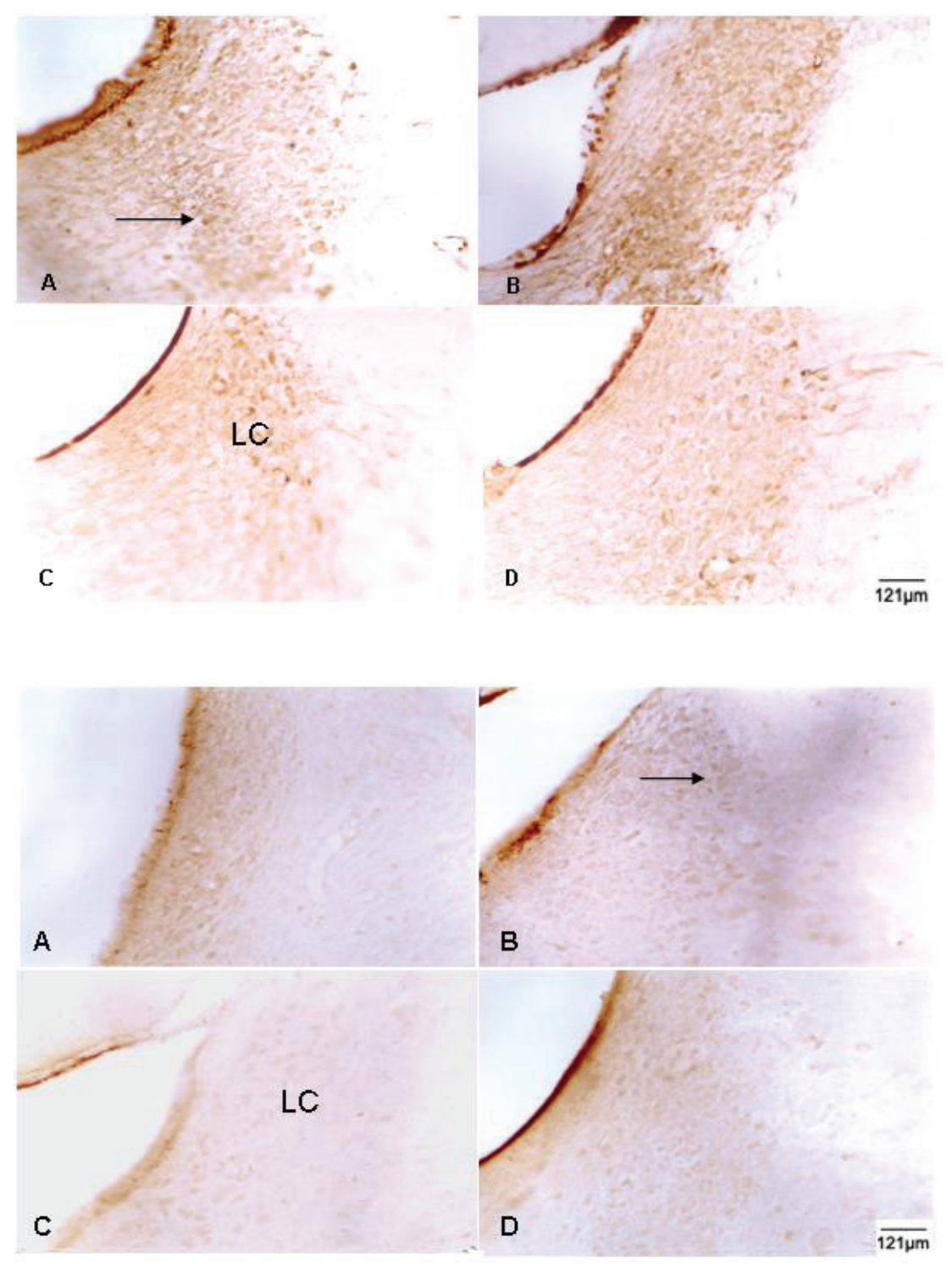

Fig. 3. Changes of necrosis degree in PCT in the ARF rats treated with GS for $48 \mathrm{~h}$ (HE staining). A. ARF+NS group; B. ARF+GS group; C. NS+NS group. Bar indicates $30.3 \mu \mathrm{m}$.

Fig. 4. Change of TH-IR in Locus coeruleus in the ARF rats treated with GS for 48 h. A. ARF+NS group; B. ARF+GS group; C. NS+NS group; D. NS+GS group. Bar indicates $121 \mu \mathrm{m}$, arrow points to TH-IR positive neurons.

Fig. 6. Change of $p$-ERK1/2-IR in Locus coeruleus in the ARF rats treated with GS for 48 h. A: ARF+NS group; B: ARF+GS group; C: NS+NS group; D: NS+GS group. Bar indicates $121 \mu \mathrm{m}$, arrow points to $p$-ERK1/2-IR positive neurons. 


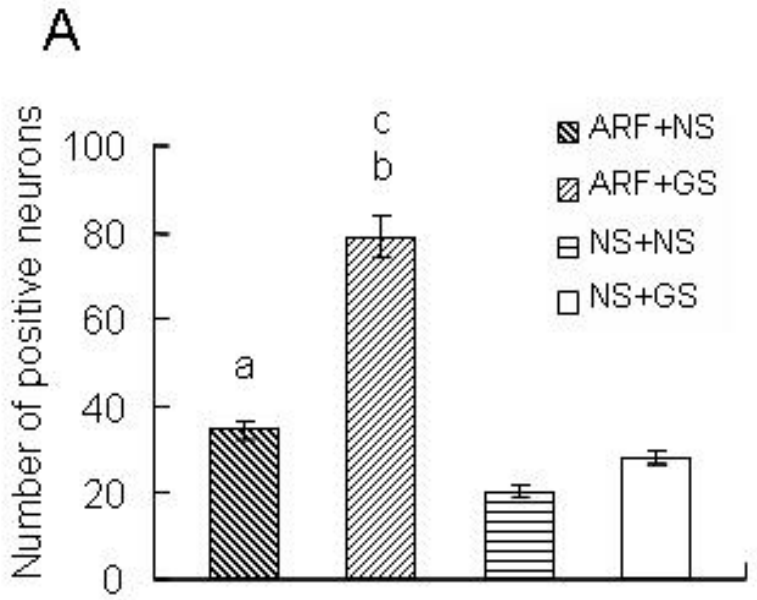

TH in the LC

\section{B}

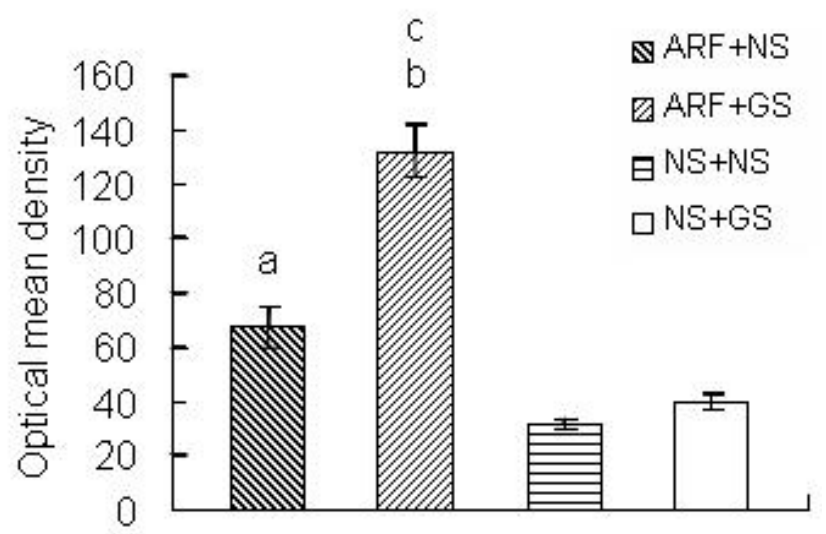

$\mathrm{TH}$ in the LC

Fig. 5. Quantitative analysis of TH-IR positive neurons and optical mean density of TH-IR in locus coeruleus in the ARF rats treated with GS for $48 \mathrm{~h}$. A. the number of TH-IR positive neurons. B. optical density of TH-IR positive neurons. Data are mean \pm S.E.M. $n=6$ ( ${ }^{\mathrm{P}} \mathrm{P}<0.05 \mathrm{ARF}+\mathrm{NS}$ group vs. NS+NS group, ${ }^{\mathrm{b}} \mathrm{P}<0.05 \mathrm{ARF}+\mathrm{GS}$ group vs. ARF+NS group, ${ }^{\mathrm{c}} \mathrm{P}<0.05 \mathrm{ARF}+\mathrm{GS}$ group vs. NS+NS group).
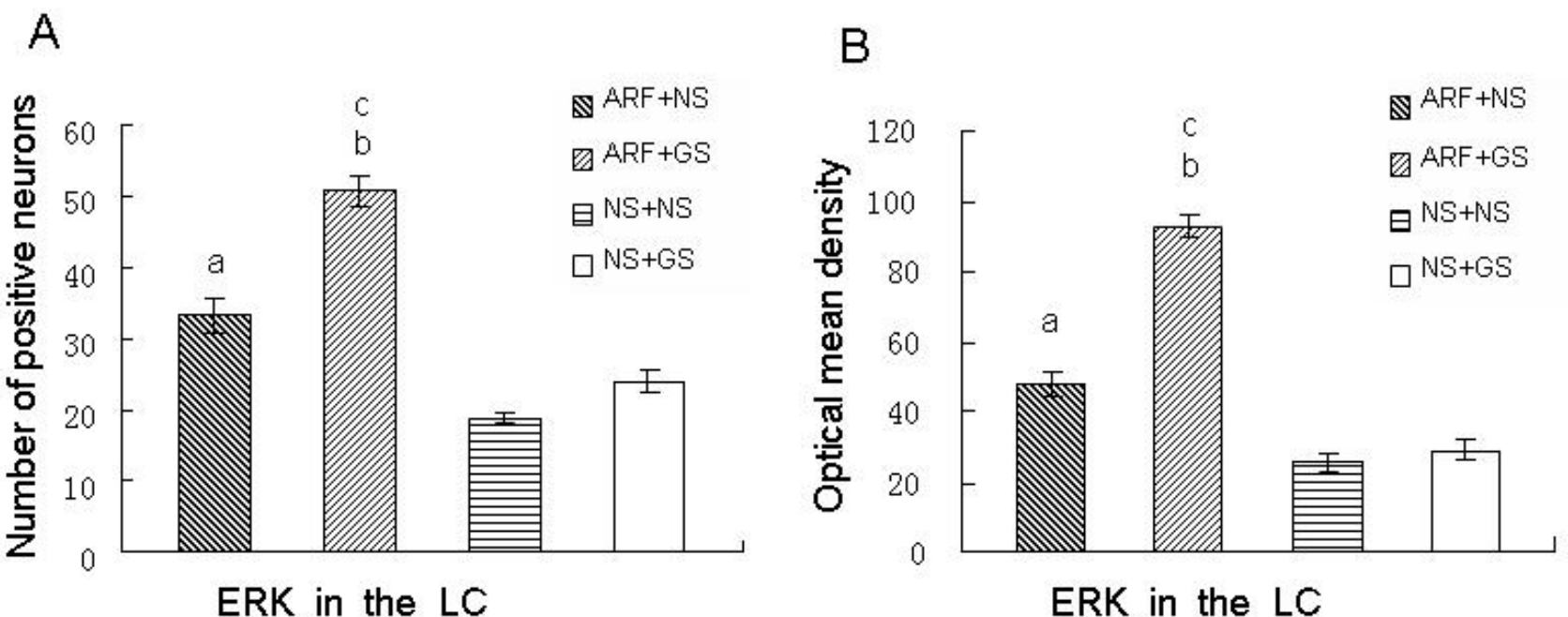

Fig. 7. Quantitative analysis of $p$-ERK1/2-IR positive neurons and optical mean density of $p$-ERK1/2-IR in locus coeruleus in the ARF rats treated with GS for $48 \mathrm{~h}$. A. the number of $\mathrm{p}$-ERK1/2-IR positive neurons. B. optical density of $\mathrm{p}$-ERK1/2-IR positive neurons. Data are mean \pm S.E.M. $n=6\left({ }^{a} \mathrm{P}<0.05\right.$ ARF+NS group vs. NS+NS group, ${ }^{b} \mathrm{P}<0.05$ ARF+GS group vs. ARF+NS group, ${ }^{c} \mathrm{P}<0.05 \mathrm{ARF}+\mathrm{GS}$ group vs. NS+NS group).

\section{Discussion}

Hypertonic glycerol injection in rats is one of the most widely used model of experimental acute renal failure (ARF). It is known as animal model of rhabdomyolysis (Abul-ezz et al. 1991). A number of studies have shown that rhabdomyolysis-induced myoglobinuric acute renal failure acounts for about $10-40 \%$ of all cases of acute renal failure (Chander et al. 2005). It has been reported that myoglobinuric acute renal failure has three pathogenic mechanisms: tubular obstruction, renal vasoconstriction and oxidative stress (Polo-Romero et al. 2004). The latter is generated through the iron released from the heme group of the myoglobin. Iron induces the formation of high-activity oxygen free radicals that increase oxidative stress and provoke lipid peroxidation and cellular death (PoloRomero et al. 2004, Vlahović et al. 2007).

Ginsenosides are triterpene saponins considered to be the main bioactive principles of the most important oriental herbal medicine "ginseng" derived from the roots and rhizomes of different Panax species (Araliaceae). Up to now more than 80 ginsenosides have been isolated from Panax species (Fuzzati 2004). Based on their structural differences, they can be classified into three categories: the panaxadiol group 
(e.g. Rb1, Rb2, Rb3, Rc, Rd, Rg3, Rh2, Rs1), the panaxatriol group (e.g. Re, Rf, Rg1, Rg2, Rh1), and the oleanolic acid group (e.g. Ro) (Tachikawa et al. 1999). The ginsenoside content of ginseng is varying depending on the Panax species, the plant age, the part of the plant, the preservation method, the season of harvest, and the extraction method.

In the present study, $48 \mathrm{~h}$ after glycerol administration the levels of BUN and Cre were significantly increased. Our data are consistent with previous reports (Chander et al. 2005). These results indicated that renal function was severely damaged in ARF rats. However, after oral administration of ginsenosides for $48 \mathrm{~h}$, the level of BUN and Cre were not increased. These results suggested that ginsenosides could obviously protect renal function of ARF rats. The morphology of glycerol-induced ARF was acute tubular necrosis, particularly in the proximal tubule. In the present study, we observed that ARF rats showed a severe acute tubular necrosis. Furthermore, we observed that the administration of ginsenosides in ARF rats relieved the severity of acute tubular necrosis. These evidences indicated that structural changes occurred in glycerol-induced ARF rats. However, oral administration of ginsenosides decreased the severity of acute tubular necrosis, and restored the renal morphology of ARF rats. The above results suggested that ginsenosides played an important part in renal protecting effect against ARF.

In glycerol-induced ARF model, reactive oxygen metabolites were proved to be the key mediators of tissue injury. Previous studies demonstrated the role of hydroxyl radical enhanced generation of hydrogen peroxide and pointed out mitochondria as a critical site of heme-induced free radical formation (Guidet et al. 1989). It was reported that ginsenosides could alleviate oxidative stress by scavenging free radicals, inhibiting of nitric oxide (NO) production which usually accompanied glutamate excitotoxicity, inducing of superoxide dismutase (SOD1) and catalase genes and reducing lipid peroxidation (Kim et al. 1998).

The malodialdehyde (MDA) content, a measure of lipid peroxidation, is paralleled with the degree of oxidative stress. Therefore, the assay of MDA could be a marker of cell damage. In the present study, we observed that MDA level in renal cortex homogenate from ARF rats was significantly increased, indicating that reactive oxidative species were generated in this model in which oxidative damage was enhanced. This was consistent with previous findings (Chander et al. 2005). In addition, we also demonstrated that the level of MDA in ARF+GS group was significantly decreased. Our results indicated that ginsenosides, as inhibitors of lipid peroxidation resulting from oxidative stress, played a crucial role in the protection against the damage associated with rhabdomyolysis.

Glutathione (GSH), a tripeptide is present in high concentrations in virtually all mammalian cells and is the most prevalent intracellular thiol. GSH has many diverse functions, one of them being the protection against oxidative damage. The importance of GSH in protecting cells against oxidative injury has been noted in numerous in vitro studies in which the depletion of GSH resulted in markedly enhanced toxicity (Suttorp et al. 1986) and increased non-protein sulfhydryl content providing a protection. We observed that the level of GSH in renal cortex homogenate of ARF rats was markedly decreased, but it obviously increased after the treatment with ginsenosides for $48 \mathrm{~h}$, indicating that intramuscular injection of glycerol produced significant depletion of renal GSH. However, oral administration of ginsenosides may alleviate oxidative stress through the increase of GSH. Taken together, ginsenosides possessed the properties of oxidative free radical scavenger, opposing lipid peroxidation production such as MDA.

The kidney is an organ richly innervated with both mechanosensitive and chemosensitive afferent nerve fibers, and renal afferent nerves project directly to a number of areas in the central nervous system contributing to arterial pressure regulation (Ciriello et al. 1983). It has been established that alterations in renal sympathetic nerve discharge with changes in neurotransmitter release could directly influence renal tubular transport function as well as renin secretion (DiBona 2005).

It has been reported that renal oxidative stress mediates the stimulation of sympathetic nerve activity in the phenol renal injury model of hypertension (Ye et al. 2006). Locus coeruleus, a pontine nucleus with high density of norepinephrine-containing neurons, also plays a role in neural regulation of cardiovascular functions, specially modulating the baroreflex (Chan et al. 1992).

It has been established that transient renal ischemia induced an increase in electrical activity of renal afferent nerve and neurons in rostroventrolateral medulla (RVLM), and enhanced Fos expression in 
RVLM neurons (Ding et al. 2001). These findings implied that renal ischemia might activate the brainstem nuclei. There was also evidence that renal ischemia could induce changes in Fos expression associated with the activation of the catecholamine-containing neurons in brainstem nuclei (demonstrated by double immunohistochemistry). This implies that catecholaminergic neurons in the brainstem nuclei could be activated by oxidative stress or renal ischemia. Recent findings showed that ginsenosides might act on the central and/or the peripheral nervous system (Nah et al. 2007). It was also reported that pretreatment with ginsenoside $\mathrm{Rg} 1$ prevented the loss of TH-positive neurons in substantia nigra in MPTP (1-methyl-4phenyl-1,2,3,6-tetrahydropyridine) - induced Parkinson mouse (van Kampen et al. 2003).

In the present study, we observed that the rats treated with GS $(\mathrm{NS}+\mathrm{GS})$ showed a slight increase of TH-IR in the LC, compared with that of NS+NS group. This result implied that in vivo ginsenosides could activate catecholaminergic neurons in the $\mathrm{LC}$ to some extent.

We observed that glycerol-induced ARF rats showed an obvious increase of TH-IR in the LC. Our results indicated that catecholaminergic neurons in the LC were excited after intramuscular injection of glycerol. This finding suggested that renal oxidative stress could enhance the expression of TH in the LC. Therefore, we hypothesized that it might be a compensatory mechanism of catecholaminergic neurons in ARF rats to renal vasoconstriction or oxidative stress. Furthermore, we also observed that the expression of TH was considerably upregulated in ARF rats treated with ginsenosides for $48 \mathrm{~h}$ compared with that in ARF+NS Group. This indicates that ginsenosides can significantly activate the catecholaminergic neurons in the LC of rats with ARF. This might be one of the effects of the ginsenosides on central nervous system in glycerol-induced ARF rats.

Other investigators have reported that several physiologically stressful stimuli, including seizure induction, ischemic insult, formalin injection and electroconvulsive shock, could activate MAPK signal in various brain regions (Imbe et al. 2004). Previous study have indicated that $\mathrm{p}$-ERK1/2 may activate $\mathrm{c}$-fos, leading to the AP-1 activation and specific TH induction in the LC in response to stress (Shimizu et al. 2004). Similarly, it has been reported that phosphorylation of both ERK1 and ERK2 was increased markedly by repeated stress.
Immunohistochemistry indicated that phospho-ERK $1 / 2$ was almost exclusively localized in the TH-positive cells of the LC following repeated stress (Hebert et al. 2005). The results of the present study also showed that p-ERK1/2-IR in the LC of ARF rats was significantly increased. It indicates that MAPK signal pathway in the LC was excited after intramuscular injection of glycerol and might be involved in the regulation of catecholaminergic neurons in the LC. In addition, we also observed that glycerol-induced ARF rats treated with ginsenosides for $48 \mathrm{~h}$ enhanced the expression of p-ERK $1 / 2$ as well as the expression of TH. Combining reported references with our results, we hypothesize that oral administration of ginsenosides might increasingly upregulate MAPK signal pathway in ARF rats, activating some related transcription factors and then regulating the catecholaminergic neurons activity. This might be one of the central mechanisms of ginsenosides against ARF.

On the basis of the above results, we propose that glycerol-induced ARF causes the changes not only in the kidney such as acute tubular necrosis and oxidative stress but also in central nervous system such as the upregulation of the TH-IR and phospho-ERK $1 / 2$-IR in the LC. We suggest that the latter should be a compensatory mechanism of central nervous system in ARF rats. Our study also shows that oral administration of ginsenosides has strong renal protective effect: restores renal histological changes, increases renal antioxidative activity by some humoral mechanisms, and improves renal function in glycerol-induced ARF rats. This study suggests a new way to be considered for the treatment of rhabdomyolysis-induced myoglobinuric acute renal failure. In addition, the possible relationship between the activation of catecholaminergic neurons in the $\mathrm{LC}$ and the renal protective effect of ginsenosides against ARF will be a focal point in our lab for the future.

\section{Conflict of Interest}

There is no conflict of interest.

\section{Acknowledgements}

This work was supported by grant No. 990211005 from the Education Department of Liaoning Province, China. The present address of Heng-ai Zhang: Institute of Materia Medica, Chinese Academy of Medical Sciences and Peking Union Medical College, Beijing, China. The present address of Min Wang: Department of Physiology, Jiaxing University, School of Medicine, Jiaxing, China. 


\section{References}

ABUL-EZZ SR, WALKER PD, SHAH SV: Role of glutathione in an animal model of myoglobinuric acute renal failure. Proc Natl Acad Sci USA 88: 9833-9837, 1991.

ANGULO JA, PRINTZ D, LEDOUX M, MCEWEN BS: Isolation stress increases tyrosine hydroxylase mRNA in the locus coeruleus and midbrain and decreases proenkephalin mRNA in the striatum and nucleus accumbens. Brain Res Mol Brain Res 11: 301-308, 1991.

CHAN JY, JANG SF, CHAN SH: Inhibition by locus coeruleus on the baroreceptor reflex response in the rat. Neurosci Lett 144: 225-228, 1992.

CHANDER V, CHOPRA K: Molsidomine, a nitric oxide donor and L-arginine protects against rhabdomyolysisinduced myoglobinuric acute renal failure. Biochim Biophys Acta 1723: 208-214, 2005.

CHANG MS, SVED AF, ZIGMOND MJ, AUSTIN MC: Increased transcription of the tyrosine hydroxylase gene in individual locus coeruleus neurons following footshock stress. Neuroscience 101: 131-139, 2000.

CIRIELLO J, CALARESU FR: Central projection of afferent renal fibers in the rat: an anterograde transport study of horseradish peroxidase. J Auton Nerv Syst 8: 273-285, 1983.

DIBONA GF: Physiology in perspective: The Wisdom of the Body. Neural control of the kidney. Am J Physiol 289: R633-R641, 2005.

DING YF, ZHANG XX, SHI GM, HE RR: Renal ischemia enhances electrical activity and Fos protein expression of rostral ventrolateral medullary neurons in rats. Sheng Li Xue Bao 53: 369-374, 2001.

FUZZATI N.: Analysis methods of ginsenosides. J Chromatogr B 812: 119-133, 2004.

GUIDET B, SHAH SV: Enhanced in vivo $\mathrm{H}_{2} \mathrm{O}_{2}$ generation by rat kidney in glycerol-induced renal filure. Am J Physiol 257: F440-F445, 1989.

HASEGAWA H, SUZUKI R, NAGAOKA T, TEZUKA Y, KADOTA S, SAIKI I: Prevention of growth and metastasis of murine melanoma through enhanced natural-killer cytotoxicity by fatty acid-conjugate of protopanaxatriol. Biol Pharm Bull 25: 861-866, 2002.

HEBERT MA, SEROVA LI, SABBAN EL: Single and repeated immobilization stress differentially trigger induction and phosphorylation of several transcription factors and mitogen-activated protein kinases in the rat locus coeruleus. JNeurochem 95: 484-498, 2005.

HOU SH, BUSHINSKY DA, WISH JB, COHEN JJ, HARRINIGTON JT: Hospital-acquired renal insufficiency: a prospective study. Am J Med 74: 243-248, 1983.

IMBE H, MURAKAMI S, OKAMOTO K, IWAI-LIAO Y, SENBA E: The effects of acute and chronic restraint stress on activation of ERK in the rostral ventromedial medulla and locus coeruleus. Pain 112: 361-371, 2004.

ISHIZUKA S, YANO T, HAGIWARA K, SONE M, NIHEI H, OZASA H, HORIKAWA S: Extracellular signalregulated kinase mediates renal regeneration in rats with myoglobinuric acute renal injury. Biochem Biophys Res Commun 254: 88-92, 1999.

KANEKO H, NAKANISHI K: Proof of the mysterious efficacy of ginseng: basic and clinical trials: clinical effects of medical ginseng, korean red ginseng: specifically, its anti-stress action for prevention of disease. J Pharmacol Sci 95: 158-162, 2004.

KANG KS, KIM HY, YAMABE N, NAGAI R, YOKOZAWA T: Protective effect of sun ginseng against diabetic renal damage. Biol Pharm Bull 29: 1678-1684, 2006.

KARIN M: The regulation of AP-1 activity by mitogen-activated protein kinases. J Biol Chem 270: 16483-16486, 1995.

KELLUM JA: What can be done about acute renal failure? Minerva Anestesiol 70: 181-188, 2004.

KIM YC, KIM SR, MARKELONIS GJ: Oh TH. Ginsenosides Rb1 and Rg3 protect cultured rat cortical cells from glutamate-induced neurodegeneration. J Neurosci Res 53: 426-432, 1998.

LAMEIRE NH, DE VRIESE AS, VANHOLDER R: Prevention and nondialytic treatment of acute renal failure. Curr Opin Crit Care 9: 481-490, 2003.

MEHTA RL, PASCUAL MT, SOROKO S, SAVAGE BR, HIMMELFARB J, IKIZLER TA, PAGANINI EP, CHERTOW GM: Spectrum of acute renal failure in the intensive care unit: the PICARD experience. Kidney Int 66: 1613-1621, 2004. 
METNITZ PG, KRENN CG, STELTZER H, LANG T, PLODER J, LENZ K, LE GALL JR, DRUML W: Effect of acute renal failure requiring renal replacement therapy on outcome in critically ill patients. Crit Care Med 30: 2051-2058, 2002.

NAH SY, KIM DH, RHIM H: Ginsenosides: are any of them candidates for drugs acting on the central nervous system? CNS Drug Reviews 13: 381-404, 2007.

NASH K, HAFEEZ A, HOU S: Hospital-acquired renal insufficiency. Am J Kidney Dis 39: 930-936, 2002.

POLO-ROMERO FJ, FERNÁNDEZ-FÚNEZ A, BROSETA VIANA L, ATIENZA MP, SÁNCHEZ GASCÓN F: Effect of $\mathrm{N}$-acetylcysteine on antioxidant status in glycerol-induced acute renal failure in rats. Renal Failure 26: 613-618, 2004.

RUSNÁK M, ZÓRAD S, BUCKENDAHL P, SABBAN EL, KVETNANSKÝ R: Tyrosine hydroxylase mRNA levels in locus ceruleus of rats during adaptation to long-term immobilization stress exposure. Mol Chem Neuropathol 33: 249-258, 1998.

SHIMIZU Y, SUGAMA S, DEGIORGIO LA, CHO BP, JOH TH: Cell-type specific signal transduction and gene regulation via mitogen-activated protein kinase pathway in catecholaminergic neurons by restraint stress. Neuroscience 129: 831-839, 2004.

SUGAYA A, YUZURIHARA M, TSUDA T, YASUDA K, KAJIWARA K, SUGAYA E: Proliferative effect of ginseng saponin on neurite extension of primary cultured neurons of the rat cerebral cortex. J Ethnopharmacol 22: 173-181, 1988.

SUN B, TANK AW: c-Fos is essential for the response of the tyrosine hydroxylase gene to depolarization or phorbol ester. J Neurochem 85: 1421-1430, 2003.

SUTTORP N, TOEPFER W, ROKA L: Antioxidant defense mechanisms of endothelial cells: glutathione redox cycle versus catalase. Am J Physiol 251: C671-C680, 1986.

TACHIKAWA E, KUDO K, HARADA K, KASHIMOTO T, MIYATE Y, KAKIZAKI A, TAKAHASHI E: Effects of ginseng saponins on responses induced by various receptor stimuli. Eur J Pharmacol 369: 23-32, 1999.

TRAVER S, MARIEN M, MARTIN E, HIRSCH EC, MICHEL PP: The phenotypic differentiation of locus ceruleus noradrenergic neurons mediated by brain-derived neurotrophic factor is enhanced by corticotropin releasing factor through the activation of a cAMP-dependent signaling pathway. Mol Pharmacol 70: 30-40, 2006.

VAN KAMPEN J, ROBERTSON H, HAGG T, DROBITCH R: Neuroprotective actions of the ginseng extract G115 in two rodent models of Parkinson's disease. Exp Neurol 184: 521-529, 2003.

VLAHOVIĆ P, CVETKOVIĆ T, SAVIĆ V, STEFANOVIĆ V: Dietary curcumin does not protect kidney in glycerolinduced acute renal failure. Food Chem Toxicol 45: 1777-1782, 2007.

WATANABE Y, MCKITTRICK CR, BLANCHARD DC, BLANCHARD RJ, MCEWEN BS, SAKAI RR: Effects of chronic social stress on tyrosine hydroxylase mRNA and protein levels. Brain Res Mol Brain Res 32: 176-180, 1995.

WEN TC, YOSHIMURA H, MATSUDA S, LIM JH, SAKANAKA M: Ginseng root prevents learning disability and neuronal loss in gerbils with 5-minute forebrain ischaemia. Acta Neuropathol 91: 15-22, 1996.

WHITMARSH AJ, DAVIS RJ: Transcription factor AP-1 regulation by mitogen-activated protein kinase signal transduction pathways. J Mol Med 74: 589-607, 1996.

YE S, ZHONG H, YANAMADALA S, CAMPESE VM: Oxidative stress mediates the stimulation of sympathetic nerve activity in the phenol renal injury model of hypertension. Hypertension 48: 309-315, 2006. 